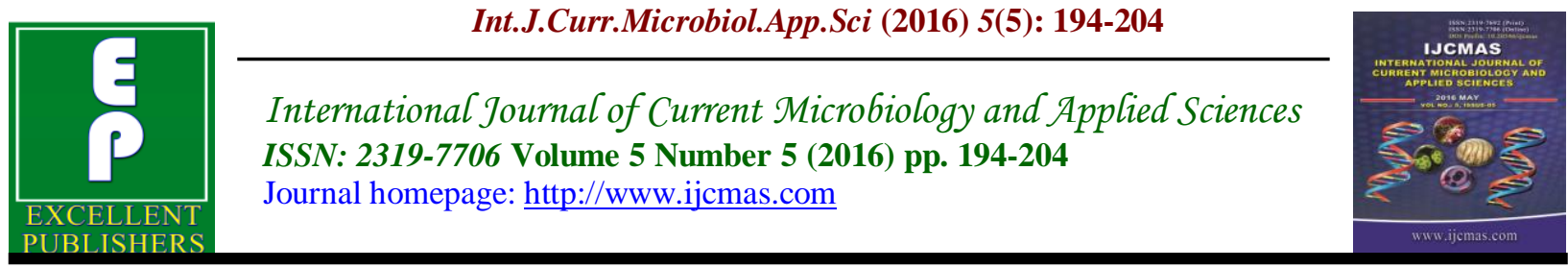

Original Research Article

http://dx.doi.org/10.20546/ijcmas.2016.505.021

\title{
Comparison of E-test and Colorimetric Micro Broth Dilution Method for Antifungal Susceptibility Testing of Candida Isolates
}

\author{
S. Prasanna ${ }^{1 *}$, Partha Roy $^{2}$, Raghu Sriram $^{3}$, Naveen Grover ${ }^{3}$, \\ Priyanka Pandit ${ }^{4}$ and Mayuri A Kulkarni ${ }^{5}$ \\ ${ }^{1}$ Department of Microbiology, Shri SathyaSai Medical College \& Research Institute, \\ Ammapettai, Kancheepuram- 608103, India \\ ${ }^{2}$ Department of Microbiology, Delhi Base Hospital, Delhi, India \\ ${ }^{3}$ Department of Microbiology, AFMC, Pune-411040, India \\ ${ }^{4}$ Department of Pathology, Command Hospital, Kolkata, India \\ ${ }^{5}$ Department of Pathology, Dr Hedgewar Hospital, Aurangabad, India \\ *Corresponding author email id:_dr_prasanna85@yahoo.com
}

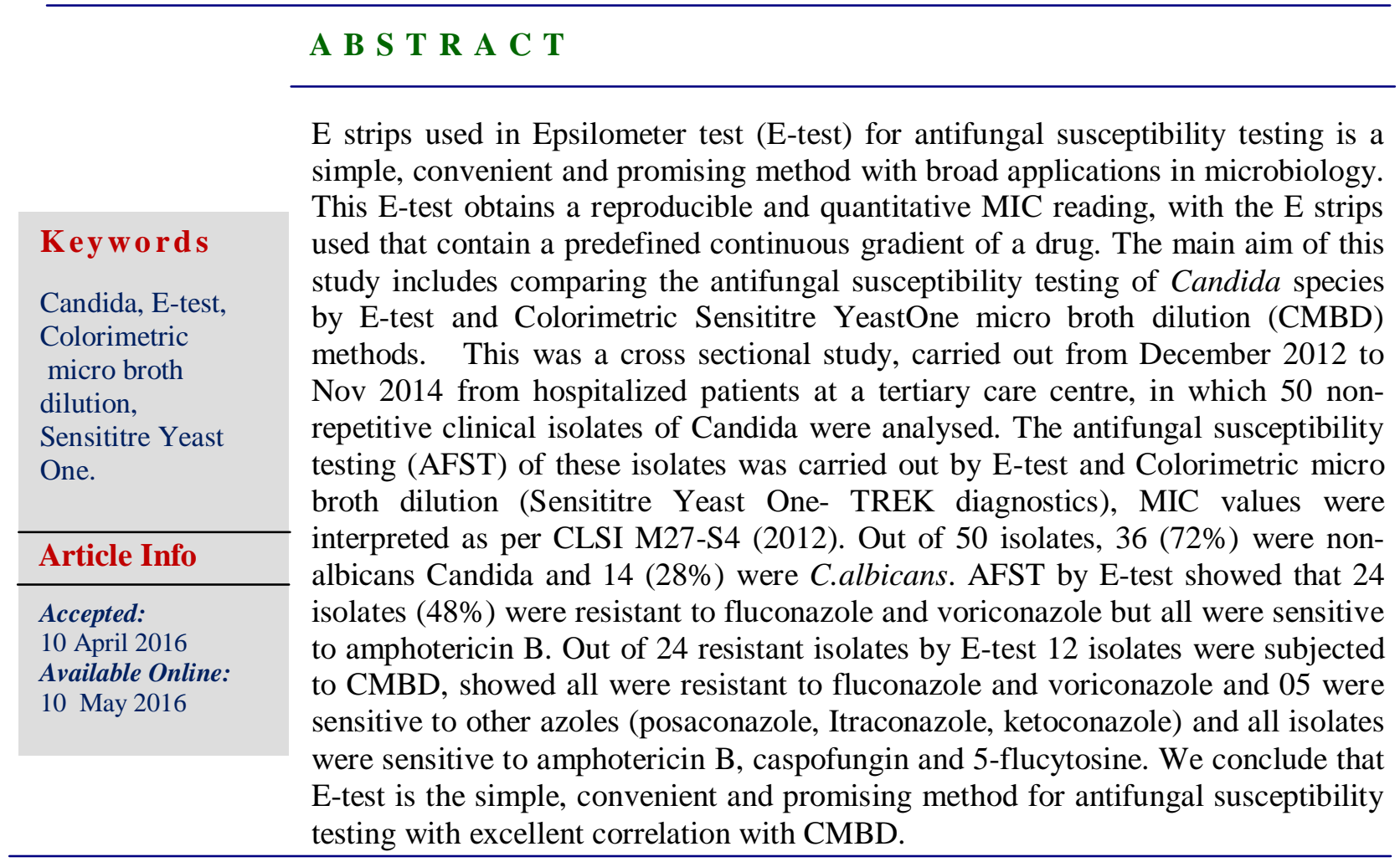

\section{Introduction}

In the past five decades there were major changes in the development of antibiotics, antineoplastic drugs, advanced intensive care support, immunomodulatory therapies, and the advent of organ transplantation increased the prognosis for many diseases. 
The significant rise in incidence of fungal infections due the result of several factors usually associated with immune dysfunction (Bodey et al., 1992; Paya, 1993). Unlike antibiotic susceptibility testing (ABST), standardized methods for antifungal susceptibility testing (AFST) are not generally available in most microbiological laboratories (Colombo et al., 1995; EspinelIngroff, 1994, 1996).

For a long period of time, fungal infections were used to be managed by azoles and other topical agents. After a long period of slow development, the last two decades has seen the introduction of an important new class of antifungal agents (the echinocandins), expansion of the spectrum of an established class of agents through chemical modification (the triazoles), and the development of lipid based formulations of amphotericin B. These developments have changed the standard of care for the treatment of many invasive fungal infections, particularly aspergillosis and candidiasis (Theodore et al., 2008).

In 1992, National Committee for Clinical Laboratory Standards (NCCLS) proposed the macro broth dilution reference for yeasts (NCCLS M27-P), then followed by M27-T in the year 1995 and NCCLS M27-A in 1997 (Theodore, 1992, 1995, 1997). The above documents are an important landmark in the field of antifungal susceptibility testing. NCCLS M27-A (1997) and NCCLS M27-A2, provides the guidelines for AFST of Candida spp and Cryptococcus neoformans and shows interpretative breakpoints in susceptible, intermediate and resistant for Candida species against azoles, flucytosine and other antifungals. NCCLS now called as Clinical and Laboratory Standard Institute (CLSI) came out with breakpoints for yeasts and yeast like fungi by micro broth dilution as the reference method (CLSI M27-A3 and M27-S4)
(Clinical and Laboratory Standards Institute (CLSI), 2008). In vitro susceptibility testing of antifungal agents by $\mathrm{E}$ test (AB Biodisk, Solna, Sweden) for fungi has met with reasonable success. This E-test obtains a reproducible and quantitative MIC reading, with the $E$ strips used that contain a predefined continuous gradient of a drug (Lozano-Chiu et al., 1998; Sanchez and Jones, 1992). In the present study, we compared Etest method and Colorimetric micro broth dilution (Sensititre Yeast OneTREK diagnostics) method by determining the MICs of clinical isolates of Candida species.

\section{Materials and Methods}

A cross sectional study was carried out from Dec 2012 to Nov 2014, in which a total of 50 non-repetitive clinical isolates of Candida were included from various clinical samples like blood, urine, muco-cutaneous samples (nail clippings, oral scrapings and high vaginal swabs), pleural fluids and continuous ambulatory peritoneal dialysis (CAPD) fluids. The blood samples were collected in BACTEC (BD BACTEC Mycosis IC/F, Ireland) and brain heart infusion (BHI) bottles under aseptic precautions. Clean catch midstream urine, urine from catheter, body fluids and CAPD dialysate were collected in wide mouthed sterile universal containers. Samples were transported and processed immediately as per standard laboratory protocols.

The organism was identified using conventional methods that included Gram stain, colony morphology in Sabouraud dextrose agar (SDA) with and without chloramphenicol, Germ tube test, CHROM agar inoculation (HI media), sugar fermentation test, sugar assimilation test and Cornmeal agar with Tween-80 inoculation (HI media). 
Antifungal susceptibility testing (AFST) was carried out by conventional methods like Etest and colorimetric micro broth dilution.

E-test: A 0.5 McFarland standard suspension of Candida strain was prepared and E-strips (AB Biodisk, Solna, Sweden) of amphotericin $\mathrm{B}$, voriconazole and fluconazole were applied over the RPMI 1640 agar with L-glutamine but without sodium bicarbonate (HI media) at $\mathrm{pH}$ 7.0. MIC of fluconazole, voriconazole and amphotericin B was determined. The zone edge intersecting the graded strip at the minimum concentration of the antibiotic was interpreted as MIC. All the results were interpreted according to CLSI 2012: M27S4.

\section{Colorimetric Sensititre Yeast One Micro Broth Dilution Method (TREK diagnostics)}

\section{Intended use}

It is a micro broth method that provides qualitative quantitative Minimum Inhibitory Concentration (MIC). The Sensititre susceptibility system is an in vitro diagnostic product for susceptibility testing of Candida species, Cryptococcus species, and Aspergillus species and miscellaneous other rapid growing yeast species.

The Sensititre yeast susceptibility test is a colorimetric micro dilution test. Each plate is dosed with antifungal agents at appropriate dilutions, and a colorimetric indicator. Results are read manually by observing the inhibition of growth at lowest antifungal concentration (as evidenced by no colour change).

\section{Inoculation Procedure}

Pick several wheel isolated colonies of $>1$ mm diameter from a pure 24 hour culture of the yeast isolate, and emulsify it into Sensititre demineralised water. Uniform suspension is required that is ensured by vortexing the mixers for 15 seconds. If clumping occurs, allow them to settle before adjusting the density. Adjust to 0.5 McFarland standard visually or using a Sensititre nephalometer. Transfer $20 \mu \mathrm{l}$ of the suspension into $11 \mathrm{ml}$ of YeastOne inoculum broth, to give an inoculum of 1.5$8 \times 10^{3} \mathrm{CFU} / \mathrm{ml}$. The step 1 and 2 should be completed within 15 minutes. Transfer 100 $\mu 1$ from YeastOne inoculum broth to the Sensititre plates within 15 minutes. Cover all wells with the adhesive seal. The plates were incubated for $24-25$ hours at $35^{\circ} \mathrm{C}$ in a non- $\mathrm{CO}_{2}$ incubator.

\section{Reading Test Results}

Using a reading mirror under normal lighting plates may be read visually, which displays the underside of the wells. Yeast growth in the antifungal solutions will be evident as a change in the colorimetric growth indicator from blue (negative) to red (positive). Some yeast may not change the indicator completely to red, but display more of a purple colour. Some organisms may show a purple colour in posaconazole, voriconazole, fluconazole, itraconazole and ketoconazole. Examine the positive growth well after 24 hours incubation (Candida species). If the growth well is still blue or only faintly purple, re-incubate for an additional 24 hours and re-examine. DO NOT READ turbidity in the Sensititre YeastOne plates. Read only colour change. The MIC is the lowest concentration of an antifungal agent that substantially inhibits growth of the organism as detected by a colour change. The amount of colour change in the wells containing the agent should be compared to the colour of the positive growth- control wells. No growth occurs 
when there is no change in the blue indicator in any dilution of an antifungal. The organism is susceptible to the lowest concentration of antifungal. The MIC is recorded as the lowest concentration of the antifungal agent without red or purple growth well, i.e. first blue. The organism is resistant to the highest concentration of antifungal when growth is seen in all wells. The MIC endpoint should be is recorded as "greater than" $(>)$ the highest concentration.

\section{Statistical Analysis}

Unpaired student's t-test was used as test of significance for quantitative variables and Chi-square test for qualitative variables. All tests were two-tailed and a $p$ value $<0.05$ was taken as significant. All tests were done using online Graph Pad software. http://www.graphpad.com/quickcalcs/contin gency2/and http://www.graphpad. com/ quickcalcs/ttest $2 /$. The concordance between E-test and CMBD methods regarding antifungal susceptibility testing of Candida isolates was measured by kappa co-efficient using http:/graphpad.com/ quickcalcs/ kappa1.cfm.

\begin{tabular}{|l|c|}
\hline $\begin{array}{l}\text { Less than chance } \\
\text { agreement }\end{array}$ & $<0$ \\
\hline Slight agreement & $0.01-0.20$ \\
\hline Fair agreement & $0.21-0.40$ \\
\hline Moderate agreement & $0.41-0.60$ \\
\hline Substantial agreement & $0.61-0.80$ \\
\hline $\begin{array}{l}\text { Almost perfect } \\
\text { agreement }\end{array}$ & $0.81-0.99$ \\
\hline
\end{tabular}

\section{Results and Discussion}

The MIC ranges as determined by both the Colorimetric micro broth dilution method and the Etest of the drugs tested against the 12 and 50 Candida isolates respectively are summarized in Table 1 and table 3 . The inoculum sizes of the original suspension used to perform the testing ranged from $1 \mathrm{x}$ $10^{6} \mathrm{CFU} / \mathrm{ml}$ to $8 \times 10^{6} \mathrm{CFU} / \mathrm{ml}$, with purity and viability confirmed by sub culturing on Sabouraud dextrose agar.

\section{E-test}

Out of 50 isolates, total of 24 isolates (12urine, 9- blood, 2- CAPD fluid and 1pleural fluid) were found to be resistant to fluconazole and voriconazole and all isolates were sensitive to amphotericin B (Fig.1, Fig.2, Table.1 and Table.2).Out 50 isolates, 24 were resistant isolates which includes: 14 C.tropicalis, 5 C.albicans, 4 C.parapsilosis and 1 C.glabrata.

\section{Colorimetric Micro Broth Dilution Method (Sensititre Yeast One- TREK Diagnostics)}

Out of these 24 resistant isolates by E-test, 12 isolates were subjected to colorimetric micro broth dilution method (Sensititre YeastOne- TREK diagnostics) for AFST in this study as the CLSI reference method (micro broth dilution). Out of 12 isolates (9blood, 1- CAPD fluid, 1- mediastinal fluid and 1- urine), all were resistant to fluconazole and voriconazole and all were sensitive to amphotericin B, caspofungin and 5-flucytosine.

Among the 12 (4 - C. tropicalis, $4-C$. parapsilosis, 3 - C. albicans, and $1-C$. glabrata) azole resistant isolates, all were resistant to fluconazole and voriconazole and 05 were sensitive to other azoles (posaconazole, Itraconazole, ketoconazole). 01 ATCC control strain was processed as control by this method, which was sensitive to all drugs (Fig.3, Fig.4 and Fig.5). The MICs for the control strain tested by the Etest method was consistently in agreement with those from the CMBD results (Table. 3 and Table.4). 
Table.1 In Vitro Susceptibility Testing of Amphotericin B, Fluconazole, Itraconazole and Flucytosine Determined by E Test for 50 Isolates

\begin{tabular}{|l|c|c|c|}
\hline Antifungal agent & $\begin{array}{l}\text { MIC Range 24 hours } \\
(\boldsymbol{\mu g} / \mathbf{m l})\end{array}$ & $\begin{array}{l}\text { MIC after 24 } \\
\text { hours }(\boldsymbol{\mu g} / \mathbf{m l})\end{array}$ & $\begin{array}{l}\text { Susceptibility/Total } \\
\text { isolates (\%) }\end{array}$ \\
\hline Fluconazole & $0.016-256$ & 0.38 & $24 / 50(48 \%)$ \\
\hline Voriconazole & $0.008-32$ & 0.032 & $24 / 50(48 \%)$ \\
\hline Amphotericin B & $0.002-32$ & 0.38 & $50 / 50(100 \%)$ \\
\hline
\end{tabular}

Table.2 Antifungal Susceptibility Pattern of Candida Isolates by E-test

\begin{tabular}{|c|c|c|c|c|c|c|}
\hline Isolates & Fluconazol & $(\%)$ & Voriconaz & le $(\%)$ & Amphoteri & $\ln B(\%)$ \\
\hline & Sensitive & Resistant & Sensitive & Resistant & Sensitive & Resistant \\
\hline C.albicans(14) & $\begin{array}{c}09 \\
(64.3 \%)\end{array}$ & $\begin{array}{c}05 \\
(35.7 \%)\end{array}$ & $\begin{array}{c}09 \\
(64.3 \%)\end{array}$ & $05(35.7 \%)$ & $14(100 \%)$ & $00(0)$ \\
\hline C.tropicalis(21) & $\begin{array}{c}07 \\
(33.3 \%)\end{array}$ & $\begin{array}{c}14 \\
(66.7 \%)\end{array}$ & $\begin{array}{c}07 \\
(33.3 \%)\end{array}$ & $14(66.7 \%)$ & $21(100 \%)$ & $00(0)$ \\
\hline $\begin{array}{l}\text { C.parapsilosis } \\
\text { (11) }\end{array}$ & $\begin{array}{c}07 \\
(63.7 \%)\end{array}$ & $\begin{array}{c}04 \\
(36.3 \%)\end{array}$ & $\begin{array}{c}07 \\
(63.7 \%)\end{array}$ & $04(36.3 \%)$ & $11(100 \%)$ & $00(0)$ \\
\hline C.glabrata (02) & $01(50 \%)$ & $01(50 \%)$ & $01(50 \%)$ & $01(50 \%)$ & $02(100 \%)$ & $00(0)$ \\
\hline $\begin{array}{l}\text { C.guilliermondii } \\
\text { (01) }\end{array}$ & $01(100 \%)$ & $00(0)$ & $\begin{array}{c}01 \\
(100 \%)\end{array}$ & $00(0)$ & $01(100 \%)$ & $00(0)$ \\
\hline C.kefyr (01) & $01(100 \%)$ & $00(0)$ & $\begin{array}{c}01 \\
(100 \%) \\
\end{array}$ & $00(0)$ & $01(100 \%)$ & $00(0)$ \\
\hline Total (50) & $26(52 \%)$ & $24(48 \%)$ & $26(52 \%)$ & $24(48 \%)$ & $50(100 \%)$ & $00(0)$ \\
\hline
\end{tabular}

Table.3 In Vitro Susceptibility Testing of Amphotericin B, Fluconazole, Ketoconazole, Itraconazole, Voriconazole, Posaconazole, Caspofungin and Flucytosine Determined By Sensititre Yeastone Colorimetric Micro Broth for 12 Isolates

\begin{tabular}{|l|c|c|c|}
\hline Antifungal agent & $\begin{array}{l}\text { MIC Range 24 hours } \\
(\boldsymbol{\mu g} / \mathbf{m l})\end{array}$ & $\begin{array}{l}\text { MIC after 24 hours } \\
(\boldsymbol{\mu g} / \mathbf{m l})\end{array}$ & $\begin{array}{l}\text { Susceptibility/Total } \\
\text { isolates }(\%)\end{array}$ \\
\hline Fluconazole & $0.125-256$ & $>256$ & $0 / 12(0)$ \\
\hline Ketoconazole & $0.008-16$ & $>8$ to $<16$ & $5 / 12(41.7)$ \\
\hline Itraconazole & $0.008-16$ & $>16$ & $5 / 12(41.7)$ \\
\hline Voriconazole & $0.008-16$ & $>16$ & $0 / 12(0)$ \\
\hline Posaconazole & $0.008-8$ & $>8$ & $5 / 12(41.7)$ \\
\hline Amphotericin B & $0.008-16$ & $>0.25$ to $<0.5$ & $12 / 12(100)$ \\
\hline Flucytosine & $0.03-64$ & $>0.06$ to $<0.12$ & $12 / 12(100)$ \\
\hline Caspofungin & $0.008-16$ & $>0.06$ to $<0.12$ & $12 / 12(100)$ \\
\hline
\end{tabular}


Table.4 Antifungal Susceptibility Pattern of Candida Species by Colorimetric Micro Broth Dilution Method (Sensititre Yeast One- TREK Diagnostics)

\begin{tabular}{|l|c|c|c|c|}
\hline \multicolumn{1}{|c|}{ Isolates } & $\begin{array}{c}\text { Fluconazole and } \\
\text { Voriconazole }\end{array}$ & \multicolumn{2}{|c|}{$\begin{array}{c}\text { All azoles } \\
\text { (PZ, FZ, IZ, } \\
\text { KZ, VOR) }\end{array}$} & \multicolumn{2}{|c|}{$\begin{array}{c}\text { Amphotericin B, Caspofungin } \\
\text { and }\end{array}$} \\
\hline & Resistant & Resistant & Sensitive & Resistant \\
\hline C.albicans $(03)$ & $00(0)$ & $03(100 \%)$ & $03(100 \%)$ & $00(0)$ \\
\hline C.tropicalis $(04)$ & $01(25 \%)$ & $03(75 \%)$ & $04(100 \%)$ & $00(0)$ \\
\hline C.parapsilosis $(04)$ & $04(100 \%)$ & $00(0)$ & $04(100 \%)$ & $00(0)$ \\
\hline C.glabrata $(01)$ & $00(0)$ & $01(100 \%)$ & $01100 \%)$ & $00(0)$ \\
\hline Total $\mathbf{( 1 2 )}$ & $05(41.7 \%)$ & $07(58.3 \%)$ & $12(100 \%)$ & $00(0)$ \\
\hline
\end{tabular}

Fig.1 Sensitive to Fluconazole and Amphotericin B (left) and Voriconazole (right)

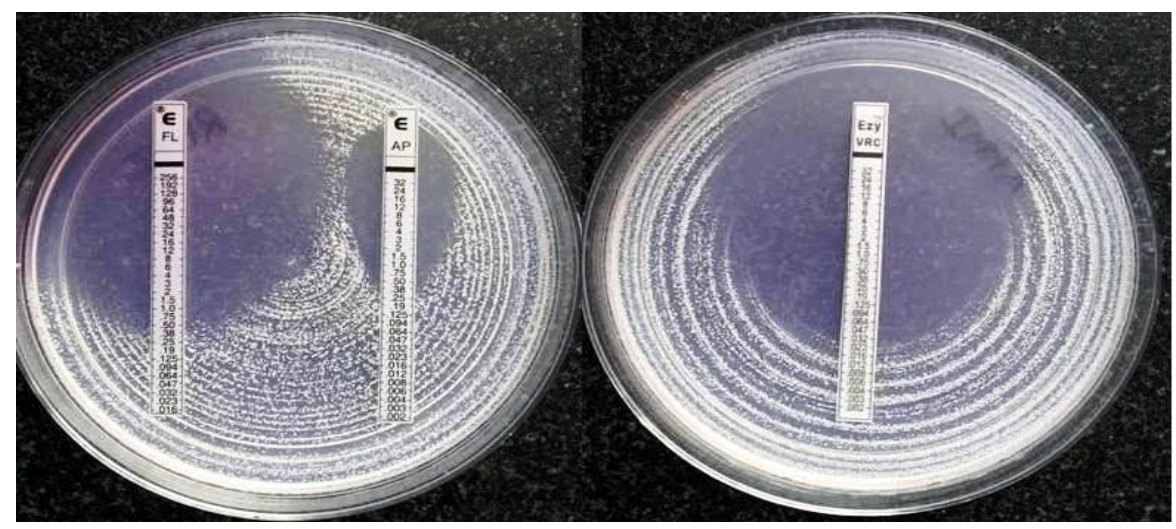

Fig.2 Resistant to Fluconazole and Voriconazole

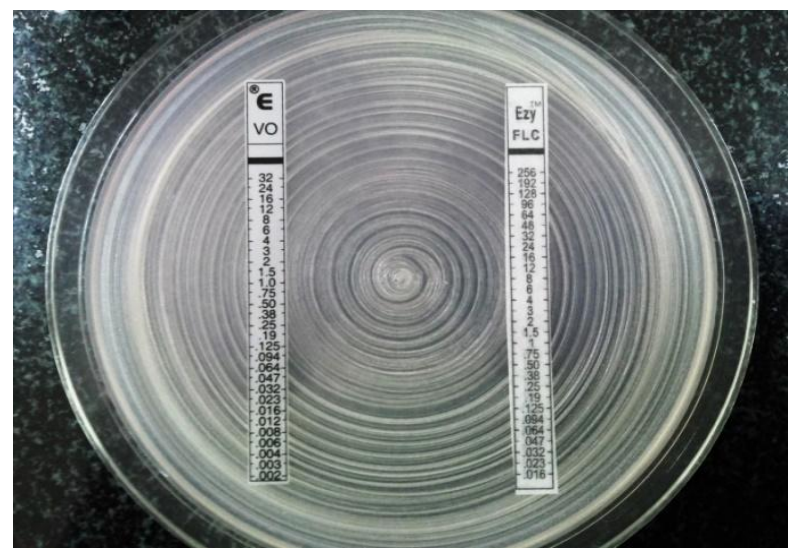


Fig.3 Sensititre Yeastone Plate Map with Various Antifungal Agents with Dilution Range

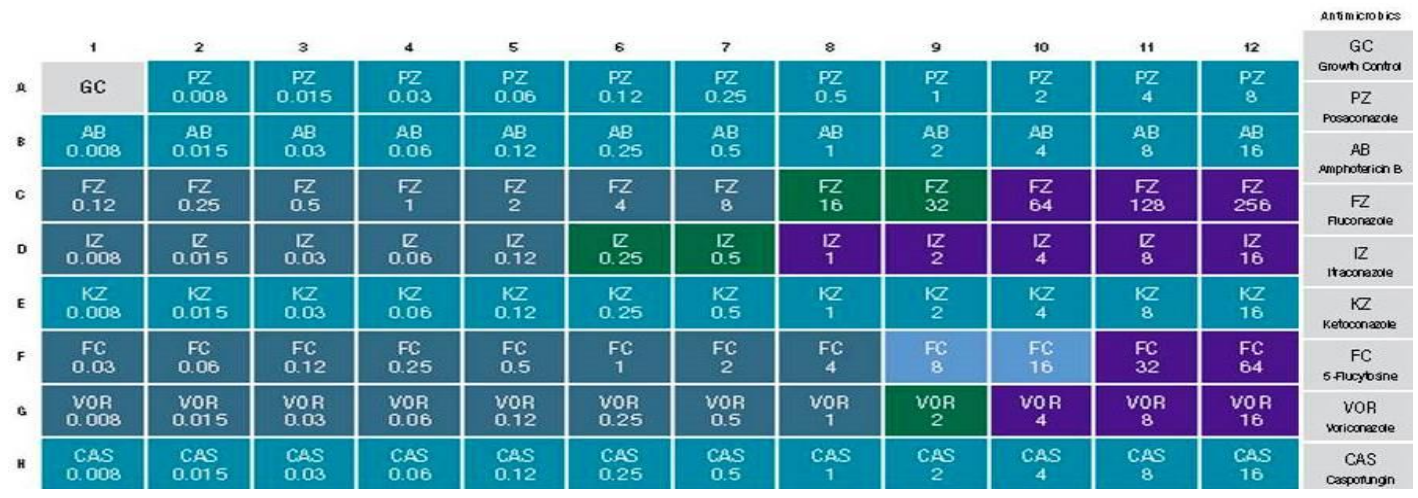

Fig.4 Sensitive to all Azoles, Amphotericin B, Caspofungin \& 5- Flucytosine

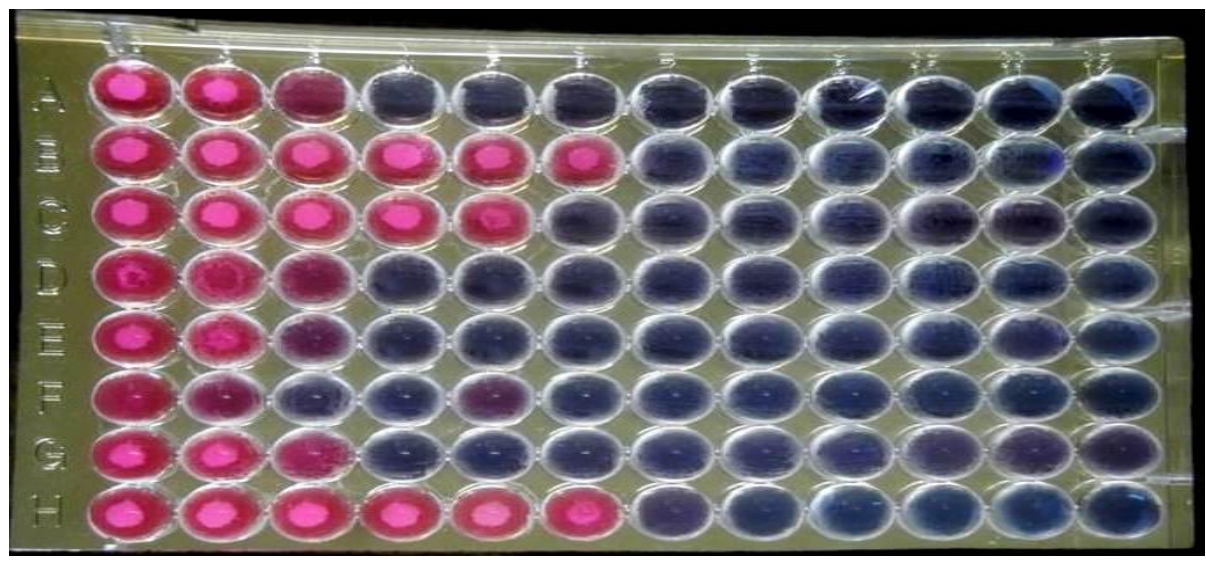

Fig.5 Resistant to all Azoles \& Sensitive (Red Arrow Head) to Amphotericin B, Caspofungin \& 5- Flucytosine

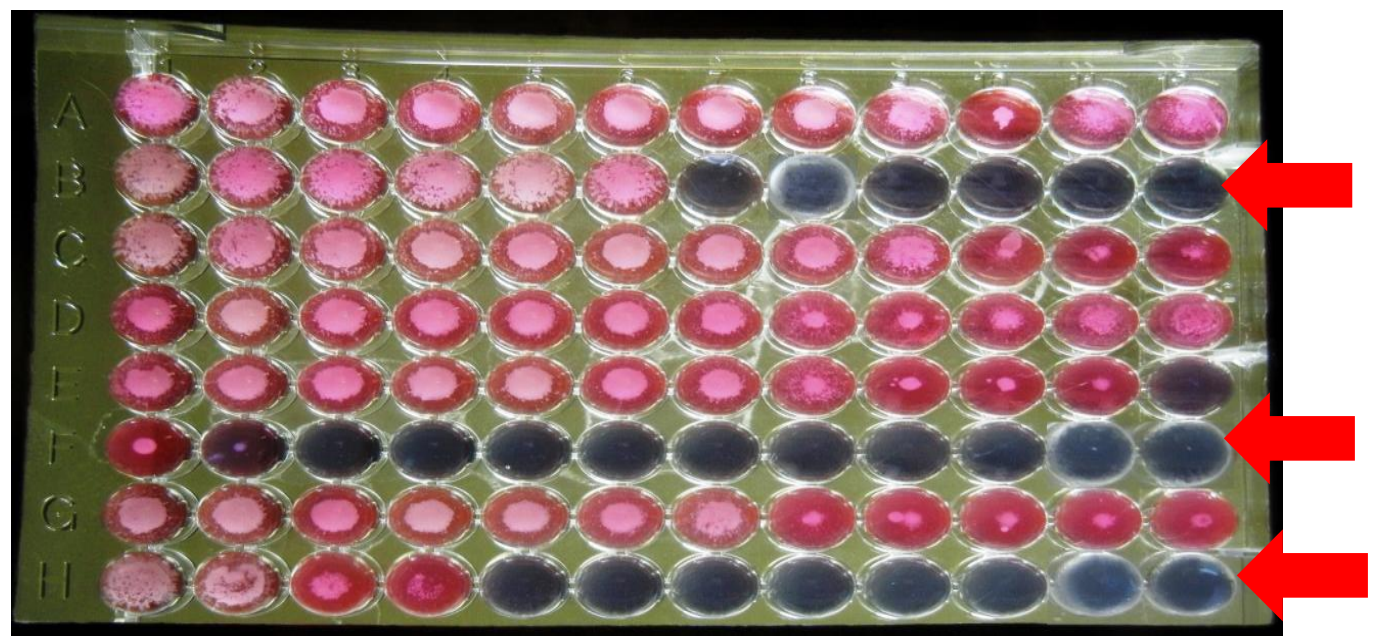




\section{Statistical Analysis}

The MIC values between the E-test method and colorimetric Sensititre YeastOne micro broth dilution method were in the range. Out of 24 resistant isolates by E-test, AFST was carried out for 12 isolates. The MIC values achieved between E-test and CMBD method showed almost perfect agreement (81-99\%) for susceptibility against fluconazole, voriconazole and amphotericin B.

Even though the NCCLS and CLSI (M27-S4 document) micro broth dilution methods improved the inter-laboratory agreement of antifungal MIC endpoint, but are not convenient method for the clinical laboratory testing. Several alternative methods have been developed when compared to the NCCLS and CLSI method for antifungal susceptibility testing. Among them, the E-test is the simple, easy for use and commercial method that has been investigated for the testing of pathogenic yeasts (Sewell et al., 1994). There are many studies carried out comparing MICs obtained by E-test with those obtained by micro or macro broth dilution have shown variable results depending of the species tested, medium and antifungal agents used. RPMI 1640 medium used in our study is the medium recommended for the susceptibility testing of yeasts and this medium improves the reproducibility of the MICs as determined by the E-test. The other studies carried out comparing between E-test and broth macro dilution methods using RPMI 1640, have showed good agreement for the azoles and flucytosine.

In this study, Out of 14 C.albicans, 09 $(64.3 \%)$ were sensitive to fluconazole and voriconazole while $05 \quad(35.7 \%)$ were resistant to both fluconazole and voriconazole and all were sensitive to amphotericin B by E-test. Out of 05 resistant C.albicans by E-test, 03 were subjected for
AFST by CMBD method Sensititre YeastOne (Y-O8). All 03 isolates were resistant to all azoles (fluconazole, itraconazole, ketoconazole, voriconazole and posaconazole) and all were sensitive to amphotericin B, Caspofungin and 5flucytosine.

Out of 21 C.tropicalis, $14(66.7 \%)$ were resistant to both fluconazole and voriconazole while $07 \quad(33.3 \%)$ were sensitive to both fluconazole and voriconazole. All were sensitive to amphotericin B by E-test. Out of 14 resistant C.tropicalis by E-test, 04 were subjected for AFST by CMBD method Sensititre YeastOne (Y-O8), of which 03 were resistant to all azoles (fluconazole, itraconazole, ketoconazole, voriconazole and posaconazole) and 01 isolate was resistant only to fluconazole and voriconazole. All isolates were sensitive to amphotericin B, Caspofungin and 5flucytosine. A study conducted by Charles et $a l$., reports the remarkable increase of azole resistance in non-albicans Candida in recent times. They suggest that newer azoles like voriconazole, posaconazole, ravuconazole and the echinocandins are suitable alternatives for such patients. In our study, isolates were even resistant to voriconazole and posaconazole (Charles et al., 2003).

Out of 11 C.parapsilosis, 07 (63.7\%) were sensitive to both fluconazole and voriconazole while $04 \quad(33.3 \%)$ were resistant to both fluconazole and voriconazole. All were sensitive to amphotericin B by E-test. Out of 04 resistant C.parapsilosis by E-test, all 04 were subjected for AFST by CMBD method Sensititre YeastOne (Y-O8), of which all were resistant only to fluconazole and voriconazole not to other azoles like posaconazole, Itraconazole and ketoconazole. All isolates were sensitive to 
amphotericin B, caspofungin and 5flucytosine.

Out of 02 C.glabrata, $01 \quad(50 \%)$ was sensitive to both fluconazole and voriconazole while $01(50 \%)$ was resistant to both fluconazole and voriconazole. All were sensitive to amphotericin B by E-test. The 01 resistant C.glabrata was subjected for AFST by CMBD method Sensititre Yeast One (Y-O8), showed resistance to all azoles (fluconazole, itraconazole, ketoconazole, voriconazole and posaconazole)and sensitive to amphotericin $\mathrm{B}$, caspofungin and 5-flucytosine. Susan et al., has stated that C.glabrata is known to possess not only innate resistance to fluconazole but rapidly acquired one as well (19).

In the present study all isolates were sensitive to amphotericin B by E-test as well as CMBD method. In other studies, they reported amphotericin $\mathrm{B}$ resistance of $6.9 \%$ and 2.5 to $16.3 \%$ (Capoor et al., 2005; Germain et al., 2001). Among the 12 isolates which were subjected for AFST by colorimetric Sensititre YeastOne micro broth dilution (Y-O8) method, all were sensitive to amphotericin $\mathrm{B}$, caspofungin and 5-flucytosine.

The azole resistant especially resistance to fluconazole, voriconazole, posaconazole mentioned in this study is most probably due to irrational use of empirical antifungal agents and predisposing risk factors like immune status of the patient, total parenteral nutrition and immunosuppressive therapies. A study conducted by MacCallum DM et al., clearly mentioned about the resistance mechanisms of in-vitro susceptibility of C.albicans to various azoles. He constructed several mutants expressing different amount of CDR efflux pumps and/or mutations in ERG11 gene encoding the target enzymes for azoles.
The present study concludes that E-test method can be a simple and convenient method for antifungal susceptibility testing with excellent correlation with colorimetric micro broth dilution method (Sensititre Yeast One). Antifungal susceptibility testing must be carried out in laboratories routinely for every Candida species apart from identification and speciation. Moreover, we cannot rely only on Vitek2 Compact for identification and antifungal susceptibility testing (AFST).

\section{Acknowledgement}

Khajuria A, Dr Deepti K, Dr Dudhat VL, Dr Nikunja Kumar Das, Dr Mugunthan M, Rao BD, Vilvanathan N, Aneesh Kumar SV and Kenjale RS.

\section{References}

Bodey, G., Bueltmann, B., Duguid, W., Gibbs, D., Hanak, H., Hotchi, M., et al. 1992. Fungal infections in cancer patients: an international survey. Eur. J. Clin. Microbiol. Infect. Dis., 11: 99-109.

Paya, C.V. 1993. Fungal infections in solidorgan transplant. Clin. Infect. Dis., 16: 677-688.

Colombo, A.L., Barchiesi, F., McGough, D.A., Rinaldi, M.G. 1995. Comparison of Etest and National Committee for Clinical Laboratory Standards broth macro dilution method for azole antifungal susceptibility testing. J. Clin. Microbiol., 33: 535-540.

Espinel-Ingroff, A. 1994. Etest for antifungal susceptibility testing of yeasts. Diagn. Microbiol. Infect. Dis., 19: 217-220.

Espinel-Ingroff, A., Pfaller, M.A., Erwin, M.E., Jones, R.N. 1996. Interlaboratory evaluation of Etest method for testing antifungal 
susceptibilities of pathogenic yeasts to five antifungal agents by using Casitone agar and solidified RPMI 1640 medium with $2 \%$ glucose. $J$. Clin. Microbiol., 34: 848-852.

Theodore, C., White, Samantha, J., Hoot. 2011. Mechanism of resistance to antifungal agents. Manual of clinical Microbiology. In Versalovic J, $10^{\text {th }}$ edition. Washington DC; ASM press. vol 2: 2008-2019.

National Committee for Clinical Laboratory Standards. 1992. Reference method for broth dilution antifungal susceptibility testing for yeasts. Proposed Standard. Document M27-P. Villanova, National Committee for Clinical Laboratory Standards.

National Committee for Clinical Laboratory Standards. 1995. Reference method for broth dilution antifungal susceptibility testing for yeasts. Tentative standard M27-T. Villanova, National Committee for Clinical Laboratory Standards.

National Committee for Clinical Laboratory Standards. 1997. Reference method for broth dilution antifungal susceptibility testing for yeasts; approved standard Document M27-A. Villanova, National Committee for Clinical Laboratory Standards. vol 17: 1-29.

National Committee for Clinical Laboratory Standards. 2002. Reference method for broth dilution antifungal susceptibility testing of yeasts: Approved standard. M27-A2 National Committee for Clinical Laboratory Standards, Wayne PA, USA.

Clinical and Laboratory Standards Institute (CLSI). 2008. Reference Method for Broth Dilution Antifungal Susceptibility Testing of Yeasts; Approved Standard. $3^{\text {rd }}$ edition. CLSI document M27-A3 (ISBN 1-56238666-2). Wayne, Pennsylvania, USA.

Clinical and Laboratory Standards Institute (CLSI). 2012. Reference Method for Broth Dilution Antifungal Susceptibility Testing of Yeasts; Approved Standard. CLSI document M27-S4. Wayne, Pennsylvania, USA.

Lozano-Chiu, M., Paetznick, V.L., Ghannoun, M.A., Rex, J.H. 1998. Detection of resistance to amphotericin B among Cryptococcus neoformans clinical isolates: performances of three different media assessed by using E-test and National Committee for Clinical Laboratory Standards M27-A methodologies. J. Clin. Microbiol., 36: 2817-2822.

Sanchez, M.L., Jones, R.N. 1992. Etest, an antimicrobial susceptibility testing method with broad clinical and epidemiologic application. Antimicrobic Newslett., 8: 1-7.

Sewell, D.L., Pfaller, M.A., Barry, A.L. 1994. Comparison of broth macro dilution, broth micro dilution, and Etest antifungal susceptibility tests for fluconazole. J. Clin. Microbiol., 32: 2099-2102.

Rodriguez-Tudela, J.L., Martinez-Suarez, J. 1994. Improved medium for fluconazole susceptibility testing of Candida albicans. Antimicrob. Agents Chemother., 38: 45-48.

Van-Eldere, J., Joosten, L., Verhaeghe, V., et al. Fluconazole and amphotericin B antifungal susceptibility testing by National Committee for Clinical Laboratory Standards broth macrodilution method compared with E-test and semiautomated broth microdilution test. J. Clin. Microbiol., 34: 842-847.

Charles, P.E., Doise, J.M., Quenot, J.P., Aube, H., Dalle, F., et al. 2003. Candidaemia in critically ill patients: 
difference of outcome between medical and surgical patients. Intensive Care Med., 29: 2162-2169.

Susan, M.N., Charles, P.C. 2003. Detection of fluconazole resistant isolates of C.glabrata by using an agar screen assay. J Clin Microbiol., 41(5): 214143.

Capoor, M.R., Nair, D., Deb, M., Verma, P.K., Srivastva, L., Aggarwal, P. 2005. Emergence of non-albicans Candida species and antifungal resistance in a tertiary care hospital. Jpn. J. Infect. Dis., 58: 344-48.

Germain, S., Lowerdiese, M., Pelletier, R., Bourgault, A.M., Libman, M., Lemieux, C., Noel, G. 2001. Prevalence and antifungal susceptibility of 442 Candida isolates from blood and other normally sterile sites: Results of a 2 year(1996-1998) multicentre surveillance study in Quebec, Canada. J. Clin. Microbiol., 39: 949-953.

Yang, Y.L., Li, S.Y., Cheng, H.H., Lo, H.J. 2002. Susceptibilities to amphotericin
B and fluconazole of Candida species in TSRAY. Diagn. Microbiol. Infect. Dis., 51: 178-83.

Chaturvedi, V., Ramani, R., Rex, J.H. 2004. Collaboration study of antibiotic medium 3 and flowcytometry for identification of Amphotericin B resistant Candida isolates. J. Clin. Microbiol., 42(5): 2252-54.

Powderly, W.G., Kobayashi, G.S., Herzig, G.P., Medoff, G. 1988. Amphotericin $B$ resistant yeast infection in severely immunocompromised patients. Am. J. Med., 84: 826-832.

MacCallum, D.M., Coste, A., Ischer, F., et al. 2010. Genetic dissection of azole resistance mechanisms in Candida albicans and their validation in a mouse model of disseminated infection. Antimicrob. Agents Chemother., 54: 1476-1483.

GraphPad Software Quickcalcs (home page on internet). No date (cited 2014 Nov 20).

\section{How to cite this article:}

Prasanna, S., Partha Roy, Raghu Sriram, Naveen Grover, Priyanka Pandit and Mayuri A Kulkarni. 2016. Comparison of E-test and Colorimetric Micro Broth Dilution Method for Antifungal Susceptibility Testing of Candida Isolates. Int.J.Curr.Microbiol.App.Sci. 5(5): 194204. doi: http://dx.doi.org/10.20546/ijcmas.2016.505.021 